\title{
Ranking of Brain MRI features for Dementia Diagnosis
}

Manasi Patil, SVERI's COE Pandharpur, Anil Yardi

\begin{abstract}
Dementia refers to the loss of memory and other cognitive skills due to changes in the brain caused by disease or trauma. An estimate tells 24 million people worldwide suffer from dementia and the numbers are growing. Many people don't understand the difference between Alzheimer's and other forms of dementia, causing many cases to go undiagnosed and untreated. The study has been carried out on various brain MRI datasets, by applying various feature extraction techniques, and the classification results are ranked in this paper.
\end{abstract}

Keywords-Brain MRI, feature extraction, classification

\section{Introduction}

Alzheimer's disease dementia, affect a growing population of elderly people today. The predictions about the course of the disease are a key component of health care decision making for patients with Alzheimer's. These predictions are difficult to make because of the high variability and nonlinearity exhibited by individual patterns of cognitive decline. Research in 2008 showed that MRI can be useful in Alzheimer's diagnosis. Scientists found that an automated system for measuring the volume of the hippocampus (region within brain) using MRI can help doctors to diagnose Alzheimer's more accurately, at an earlier stage. MRI also helped researchers identify abnormal structural changes in the brains of seemingly normal elderly individuals that aided detection of mild cognitive impairment, a potential precursor to Alzheimer's disease. In addition, researchers found that MRI scans that detect shrinkage in specific regions of the mid-brain accurately diagnosed neurodegenerative disease such as Alzheimer's even before symptoms interfere with memory. The role of artificial neural networks in medical diagnosis, prognosis, treatment and clinical decision support has been well established since the earliest days of computing.

In summary, the research conducted, improves the current Dementia classification techniques in which the task is to extract patterns from brain MR images and build accurate and transparent classifiers using artificial neural networks.

\section{Ranking of Results}

\section{A. 3D MRI from ADNI Database}

Table 1: Performance of various feature extraction methods:Accuracy

\begin{tabular}{|c|c|}
\hline $\begin{array}{c}\text { Feature Extraction } \\
\text { Method }\end{array}$ & Accuracy(3class) \\
\hline $\begin{array}{c}\text { Volume }+ \\
\text { Demographic }\end{array}$ & $90 \%$ \\
\hline $\begin{array}{c}\text { VBM Volume } \\
\text { +Affine coefficients }\end{array}$ & $90.19 \%$ \\
\hline Spatial Texture & $98 \%$ \\
\hline $\begin{array}{c}\text { VOI } \\
\text { intensity(2class) }\end{array}$ & $86 \%$ \\
\hline Freq filter-spatial & $100 \%$ \\
\hline DWT & $83 \%$ \\
\hline
\end{tabular}

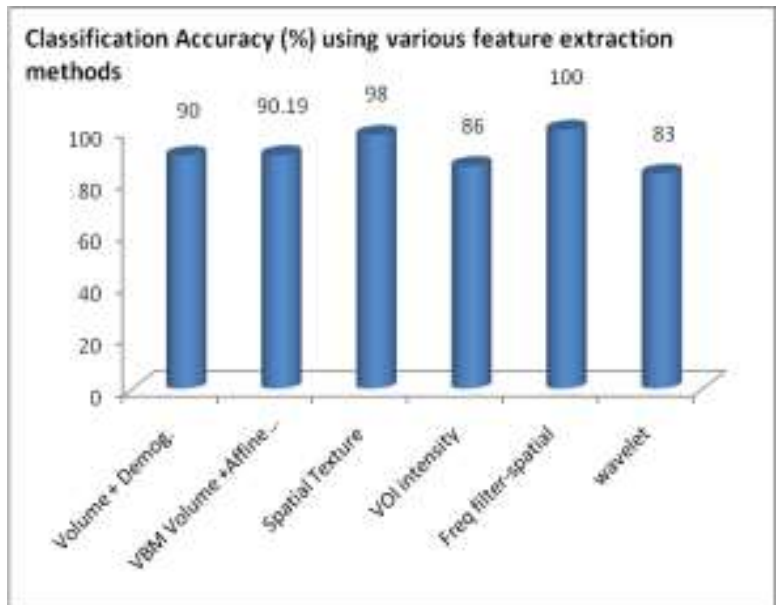

Figure 1: Performance Comparison-

Table 2: Performance of various feature extraction methods: (Processing time) Epochs

\begin{tabular}{|c|c|}
\hline $\begin{array}{c}\text { Feature Extraction } \\
\text { Method }\end{array}$ & Epochs \\
\hline $\begin{array}{c}\text { Volume + } \\
\text { Demographic }\end{array}$ & 600 \\
\hline $\begin{array}{c}\text { VBM Volume +Affine } \\
\text { coefficients }\end{array}$ & 2851 \\
\hline Spatial Texture & 191 \\
\hline VOI intensity & 1000 \\
\hline Freq filter-spatial & 62 \\
\hline DWT & 6000 \\
\hline
\end{tabular}


Proc. of The Fifth Intl. Conf. On Advances in Computing, Electronics and Electrical Technology - CEET 2016 Copyright (C) Institute of Research Engineers and Doctors, USA .All rights reserved.

ISBN: 978-1-63248-087-3 doi: 10.15224/ 978-1-63248-087-3-13

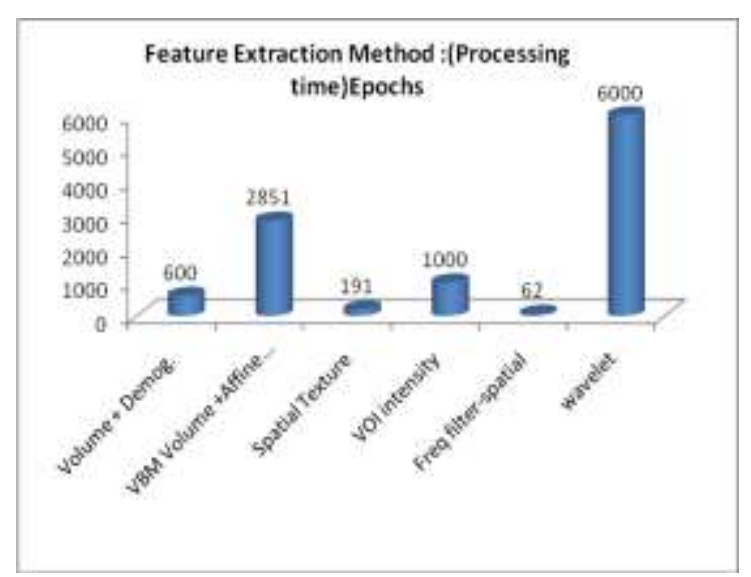

Figure 2: Performance Comparison-

Processing Time

\section{B. Experiments on 2D MRI from Whole brain atlas Database}

Table 2: Performance of various Feature extraction methods

\begin{tabular}{|c|c|}
\hline $\begin{array}{c}\text { Feature } \\
\text { Extraction } \\
\text { Method }\end{array}$ & Accuracy(3class) \\
\hline DWT features & $97.2 \%$ \\
\hline $\begin{array}{c}\text { Spatial features } \\
\text { of frequency } \\
\text { filtered image }\end{array}$ & $100 \%$ \\
\hline Slantlet features & $100 \%$ \\
\hline DCT features & $100 \%$ \\
\hline
\end{tabular}

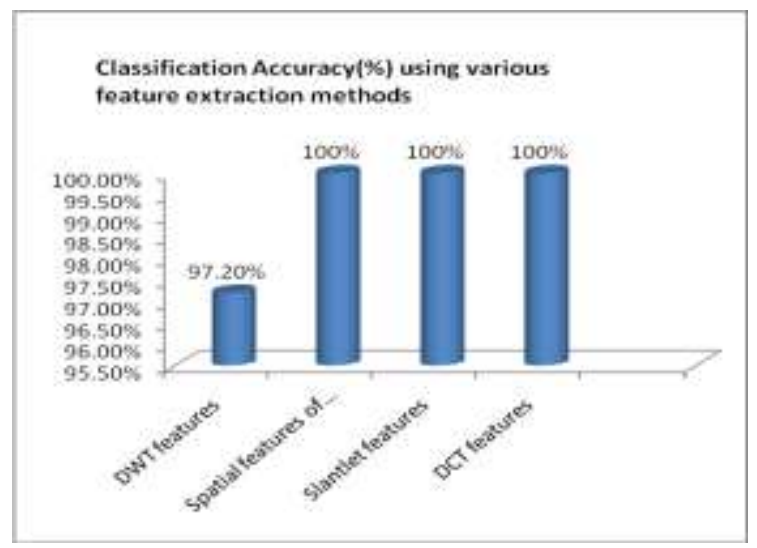

Figure 3: Performance of various Levels of classifier
Table 3: Performance of various feature extraction methods: (Processing time) Epochs

\begin{tabular}{|c|c|}
\hline $\begin{array}{c}\text { Feature Extraction } \\
\text { Method }\end{array}$ & Epochs \\
\hline DWT features & 1726 \\
\hline $\begin{array}{c}\text { Spatial features of } \\
\text { frequency filtered } \\
\text { image }\end{array}$ & 351 \\
\hline Slantlet features & 39 \\
\hline DCT features & \\
\hline
\end{tabular}

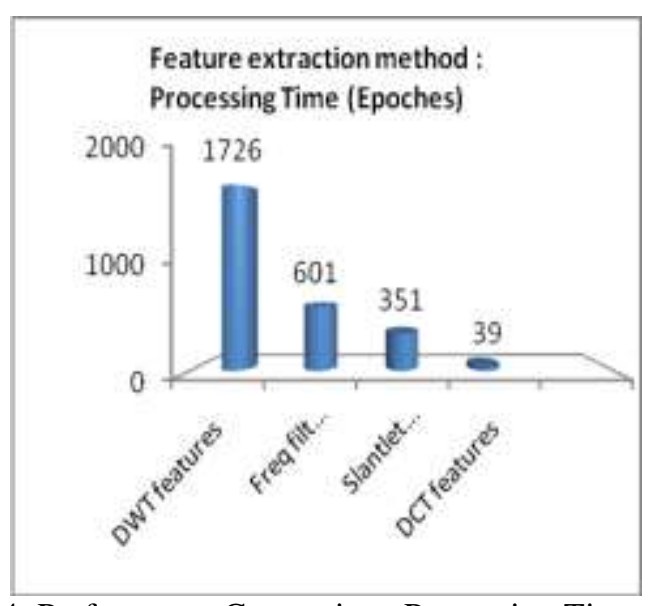

Figure 4: Performance Comparison-Processing Time

\section{Conclusion and Discussion}

The primary objective of this research was to develop methods and algorithms to identify or classify types of neurodegenerative diseases; which is fulfilled by investigating and improving feature extraction methods on the neurodegenerated brain image. These features are then used as the inputs to the artificial neural networks and successfully classified the type of Dementia. Particular achievements are

i) Identified database ADNI with sufficient number of $\mathrm{MR}$ images of $\mathrm{AD}, \mathrm{MCI}$ and normal. As ADNI is not open access, obtained access to this database by submitting research rationale to $\mathrm{ADNI}$. 
Proc. of The Fifth Intl. Conf. On Advances in Computing, Electronics and Electrical Technology - CEET 2016 Copyright (C) Institute of Research Engineers and Doctors, USA .All rights reserved.

ISBN: 978-1-63248-087-3 doi: 10.15224/ 978-1-63248-087-3-13

b) Downloaded and successfully installed SPM and VBM toolkit in MATLAB, for preprocessing of 3D MR images of brain.

c) Voxel based morphometry (VBM) for normalization of $3 \mathrm{D}$ brain images and finding brain tissue volumes.

d) Did first classification attempt using brain tissue volumes and affine coefficients (VBM normalization), as features, subsequent ANN was $90 \%$ successful.

e) Secondly did VOI analysis for feature extraction from hippocampus (part inside brain) and ANN classified with $86 \%$ accuracy.

f) Extraction of texture features from brain image and design ANN classifier resulted into $98 \%$ accuracy.

g) Method of applying frequency filter to brain image and obtaining spatial features and ANN classification resulted the best, gave $100 \%$ accuracy.

h) Also attempted to get DWT coefficients of 3D MRI as features for ANN input, but could not give accuracy above $77 \%$.

i) Downloaded 2D axial MR images belonging to six classes from Whole brain atlas database. Applied DWT method to get $97.2 \%$ accuracy.

j) Used frequency filtering method (described in 7 above) to these 2D MR images and could get $100 \%$ classification accuracy up to 3 classes.

k) Keeping belief in multiresolution analysis an attempt was made to extract Slantlet transform features from 2D MRI and achieved success with $100 \%$ accuracy even for 4 class classification.

1) Used Discrete Cosine Transform based features and a robust classifier with $100 \%$ accuracy, even up to five classes was obtained.

\section{Iv. Future Scope}

Dementia classification can be taken towards more detail categories subject to the availability of Neuroimaging databases with sufficient number of cases of each and every disease type. Further work can be done to establish statistical significance through the use of numerous training sets as and when the databases get available with sufficient number samples.

Also according to the opinion from Neurologist, results of this research if combined with other clinical data, like onset of the disease or history it may lead to more robust diagnosis system.

\section{Acknowledgment}

Authors are thankful to Alzheimer's Disease Neuroimaging Initiative (ADNI) database (www.loni.ucla.edu/ADNI). Authors are also thankful to the Whole brain atlas database at http:// www.med.harvard.edu/ AANLIB/nav.htm., for the images.

\section{References}

[1]http://www.helpguide.org/elder/alzheimers_disease_sym ptoms_stages.htm

[2] Helen Chiu Culture and Dementia From Diversity to Partnership Department of Psychiatry The Chinese University of Hong Kon Alziemers association, the year in alzheimer science 2008 Strides in Early Detection of Alzheimer's Disease W. Selesnick, The slantlet transform, IEEE Trans on Signal Processing, vol 47, no 5, pp1304-1313, May 1999.

[3] W. Selesnick, The slantlet transform, IEEE Trans on Signal Processing, vol 47, no 5, pp 1304-1313, May 1999.

[4] Madhubanti Maitra, Amitava Chatterjee, A Slantlet transform based intelligent system for magnetic resonance brain image classification, Biomedical Signal Processing $\begin{array}{llr}\text { and } & \text { Control 299-306 }\end{array}$ doi:10.1016/j.bspc.2006.12.001

[5] http://www.med.harvard.edu/AANLIB/nav.htm. [6] R.K. Begg, M. Palaniswami, B. Owen, Support vector machines for automated gait classification, IEEE Biomed. Eng. 52 (5) (2005) 28-838.

About Author (s):

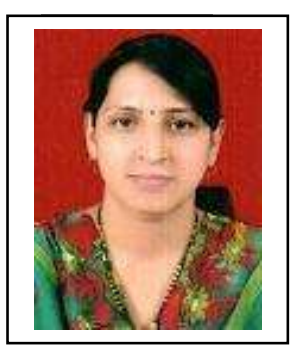

[Dr. Mrs. M. M. Patil, is a Assoc. Professor in Electronics and Telecom Dept. of SVERI's College of Engg. Pandharpur. She did her graduation in Industrial Electronics from PVPIT Sangli in 1998 post graduation in Electronics from WCE Sangli in 2006, $\mathrm{Ph}$.D. from WCE Sangli in 2013. She has to her credit 25 publication. She has guided numerous students for graduation and Post graduation. She is a life member of Indian Society for Technical Education. Her areas of interest are Image Processing, Pattern Recognition..]

Prof A. R. Yardi, was a Professor in Electronics and Deputy Director at Walchand College of Engineering Sangli,(India). He did his graduation in Electronics and Telecommunications Engineering, from College of Engineering, Pune in 1971 and post graduation in Electrical Engineering from Indian Institute of Technology, Mumbai, in 1975. He has to his credit a vast publication of about 50 papers in international, national journals and conferences. He has guided numerous students for Post graduation and for Ph.D.in Electronics 